\title{
Solar photospheric magnetic reconnection
}

\author{
Akitsugu Takeuchi ${ }^{1}$ and Kazunari Shibata ${ }^{2}$ \\ ${ }^{1}$ Yonago National College of Technology, Hikona 4448, Yonago, Tottori 683-8502, Japan \\ ${ }^{2}$ Kwasan Observatory, Kyoto University, Yamashina, Kyoto 607-8471, Japan
}

(Received May 30, 2000; Revised October 30, 2000; Accepted February 28, 2001)

\begin{abstract}
We investigate photospheric magnetic reconnection due to an encounter of oppositely directed vertical magnetic flux sheets, performing 2.5-dimensional magnetohydrodynamic (MHD) numerical simulations. We construct the initial flux sheets adopting the thin flux tube approximation. Since actual solar resistivity possesses a maximum at the temperature-minimum region, we adopt a resistivity model in which the resistivity is described as a function of height with a maximum (where the magnetic Reynolds number $=1000$ ) at a middle height of our simulation box. Owing to the resistivity, the Sweet-Parker type reconnection occurs at the middle. The inflow speed $\left(v_{i} \sim 160\right.$ $\mathrm{m} / \mathrm{s}$ ) is nearly equal to the speeds implied by observations of canceling magnetic features on the photosphere. Thus photospheric reconnection seems to be a cancellation mechanism. It is shown that upward propagating MHD slow mode waves are generated by an upward reconnection jet. Moreover, when we incline the initial field lines $30^{\circ}$ from the vertical direction in the other flux sheet, Alfvén waves are also generated as a result of the reconnection. The energy flux carried by the slow modes and Alfvén waves are $10^{10}$ and $10^{8} \mathrm{erg} / \mathrm{cm}^{2} / \mathrm{s}$, respectively, and the durations are $40 \mathrm{~s}$. Since in models of solar spicules upward propagating slow waves or Alfvén waves are usually assumed as the initial perturbations, we compare the energy of both waves. It is found that the wave energies due to the reconnection are comparable to those assumed in spicule models. Thus the photospheric magnetic reconnection might be one of the causes of solar spicules.
\end{abstract}

\section{Introduction}

Recently using SoHO MDI, Schrijver et al. (1997, 1998) found that in 1.5 to 3 days as much magnetic flux is cancelled as is present in quiet-network elements. The rate of flux cancellation is $\sim 3 \times 10^{21} \mathrm{Mx} / \mathrm{hr}$ on the entire Sun, which is one order of magnitude bigger than that found with previous ground based observations. The discovery stimulates us to investigate photospheric magnetic reconnection theoretically (Litvinenko, 1999; Sturrock, 1999). Recently, performing magnetohydrodynamic (MHD) numerical simulations, Takeuchi and Shibata (2001) studied photospheric reconnection induced by convective intensification of solar surface magnetic fields. However, they could not discuss the reconnection point precisely, because they assumed a uniform resistivity in their model. Actual solar resistivity strongly depends upon the height in the atmosphere and it possesses a maximum at the temperature-minimum region. Thus, we investigate the photospheric reconnection due to an encounter of oppositely directed vertical magnetic flux sheets, by adopting a resistivity model in which the resistivity is a function of height with a maximum (where magnetic Reynolds number $=1000$ ) at a middle height of our simulation box, performing 2.5-dimensional MHD numerical simulations.

In the following section we present the basic assumptions adopted in our calculations. In Section 3 we present our

Copy right $(\mathrm{C}$ The Society of Geomagnetism and Earth, Planetary and Space Sciences (SGEPSS); The Seismological Society of Japan; The Volcanological Society of Japan; The Geodetic Society of Japan; The Japanese Society for Planetary Sciences. results and discuss them. In final section we summarize our results.

\section{Models}

We solve the resistive MHD equations numerically in a two-dimensional (2D) Cartesian geometry $(x, z)$ where the $z$-axis is directed vertically upwards to the solar surface. In the calculations the $y$-components of vectors are assumed as a function of $x$ and $z$. We assume that the fluid is an ideal gas with no viscosity, and adopt the adiabatic energy equation in which $\gamma$ (the ratio of specific heats) is taken to be 5/3. The initial state is shown in Fig. 1. Widths of our simulation box, $L_{x}$ and $L_{z}$, are 1.0 and 8.0, respectively, where the unit of length is the photospheric pressure scale height $H_{p}(=160 \mathrm{~km})$. The level at $z=0$ corresponds to the photospheric base. Since oppositely directed magnetic flux sheets come into contact with each other on the $y z$-plane, the plane is a current sheet. The initial density and pressure distributions in the outside of the flux sheets are calculated assuming hydrostatic equilibrium, where the temperature is taken to be a constant $\left(T_{0}=6300 \mathrm{~K}\right)$.

We calculate the initial field configuration adopting the thin flux tube approximation (Roberts and Webb, 1978) in which the field strength is found from the pressure difference between the outside and the inside of the flux sheets. Thus, the maximum field strength in the flux sheet $B_{00}$ is calculated from

$$
B_{00}=\sqrt{\frac{8 \pi P_{e}}{1+\beta}},
$$




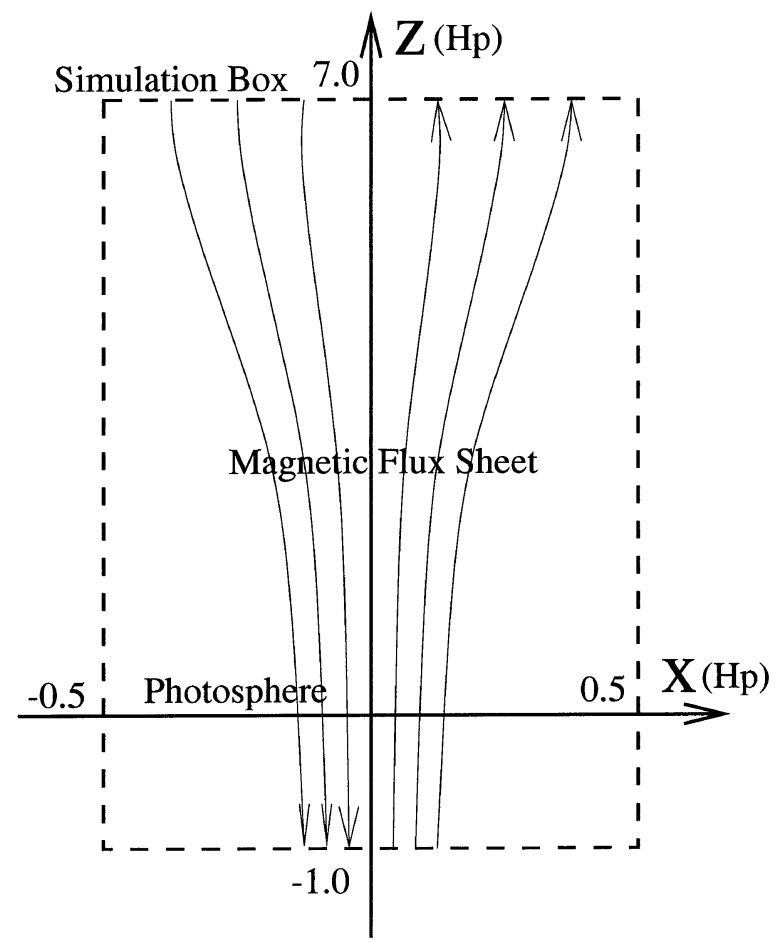

Fig. 1. Initial state of our simulations is shown. Widths of our simulation box, $L_{x}$ and $L_{z}$, are 1.0 and 8.0, respectively, where the unit of length is the photospheric pressure scale height $H_{p}(=160 \mathrm{~km})$. The level at $z=0$ corresponds to the photospheric base. Since oppositely directed magnetic flux sheets come into contact on the $y z$-plane, the plane is a current sheet.

where $P_{e}$ is the gas pressure in the outside of the flux sheets, and $\beta=p_{\text {gas }} / p_{\text {mag }}=0.3$. Using $B_{00}$ we describe the variation of the field strength $B_{0}$ in $x$-direction in the flux sheets as follows:

$$
B_{0}= \begin{cases}B_{00} \sin (\pi x / W), & \text { for } 0.5<x / W<1.0 \\ B_{00}, & \text { for } 0.2<x / W<0.5 \\ B_{00} \sin (2.5 \pi x / W), & \text { for }-0.2<x / W<0.2 \\ -B_{00}, & \text { for }-0.5<x / W<-0.2 \\ B_{00} \sin (\pi x / W), & \text { for }-1.0<x / W<-0.5\end{cases}
$$

Moreover, the initial $x, y$, and $z$-components of the magnetic fields are

$$
\begin{aligned}
& B_{z}= \begin{cases}B_{0}, & \text { for } 0.0<x / W<1.0 \\
B_{0} \cos \theta, & \text { for }-1.0<x / W<0.0\end{cases} \\
& B_{y}= \begin{cases}0, & \text { for } 0.0<x / W<1.0 \\
B_{0} \sin \theta, & \text { for }-1.0<x / W<0.0\end{cases} \\
& B_{x}=B_{z} \frac{x}{W} \frac{d W}{d z}, \quad \text { for }-1.0<x / W<1.0
\end{aligned}
$$

where $W$ is the initial width of the flux sheet. Since $B_{00}$ decreases with height and $B_{00} W$ is a constant, $W$ increases with height. At the photoshere we choose $W=0.05(8 \mathrm{~km})$. The width is smaller than the diameter of an actual photospheric flux tube. However, in the thin flux tube approximation when we decrease the width of the flux sheet the initial field configuration tends to a magnetostatic equilibrium solution. Thus the width is an optimal choice. Note that the initial field lines in the left flux sheet were inclined at an angle $\theta$ from the vertical direction, because it seems natural to assume the angle rather than to assume perfectly anti-parallel flux sheets. In the calculations we examine two cases, $\theta=0^{\circ}$ and $30^{\circ}$.

It is well known that actual solar resistivity possesses a maximum at the temperature-minimum region. To model the resistivity distribution we adopt

$$
R_{m}=\frac{H_{p} C_{s}}{\eta}=10^{3} \exp (z-2)^{2},
$$

where $R_{m}$ is the magnetic Reynolds number, $C_{s}(=8 \mathrm{~km} / \mathrm{s})$ the photospheric sound velocity, and $\eta$ the magnetic diffusivity. We evaluate $R_{m}$ using classical resistivity (Kovitya and Cram, 1983), to be $R_{m} \sim 10^{5}$ and $10^{4}$ at the photosphere and the temperature-minimum region, respectively. However, the actual current sheets between oppositely directed vertical flux tubes are fluted by convective motions. Since fluting increases the area of the current sheet, magnetic flux reconnects more quickly than in the plane current sheet case. In our 2D models, we take into account the fluting effect by decreasing $R_{m}$. Thus our choice of $R_{m}$ is probably adequate.

Owing to the maximum of the resistivity, magnetic reconnection occurs in the neighborhood of the maximum. We follow the evolution by using the modified Lax-Wendroff scheme (Rubin and Burstein, 1967), adopting an artificial viscosity (Richtmyer and Morton, 1967). In the calculations the computational grid $\Delta z$ is taken to be 0.05 . On the other hand $\Delta x$ is taken as follows. In the current sheet $(|x|<0.1$, see Fig. 1), $\Delta x$ is taken to be 0.001 . Outside the current sheet, $\Delta x$ is increased with distance from the sheet until it reaches 0.01 . If it reaches 0.01 , it is set to be 0.01 . To ensure numerical stability, the time step is chosen sufficiently small to satisfy the Courant-Friedrichs-Lewy criterion. We adopt symmetric boundary conditions at all boundaries of the simulation box.

\section{Results and Discussion}

In Fig. 2 we show temporal evolution of temperature distribution due to the magnetic reconnection, where $\theta$ is taken to be $0^{\circ}$. The horizontal and vertical directions are $x$ and $z$ directions, respectively. The unit of time and temperature are $H_{p} / C_{s}(=20 \mathrm{~s})$ and the initial photospheric temperature $T_{0}(=6300 \mathrm{~K})$, respectively. Since the resistivity possesses a maximum at $z=2$, magnetic reconnection occurs in the neighborhood of the maximum $(t=1)$. The reconnection point, however, is somewhat below the maximum, because the width of the initial current sheet increases with height. The reconnection is the Sweet-Parker type (Sweet, 1958; Parker, 1963). The width of the initial flux sheet, $W_{r}$, at the reconnection location is $0.1(=16 \mathrm{~km})$. The duration of the reconnection $\tau_{r}$ is $5(=100 \mathrm{~s})$. Thus, the inflow speed $v_{i}$ is $W_{r} / \tau_{r}=0.16 \mathrm{~km} / \mathrm{s}$. An example of the canceling features implies $v_{i} \sim 45 \mathrm{~m} / \mathrm{s}$ (Garcia de la Rosa et al., 1989). Since the inflow speed is nearly equal to the speed implied by observations of canceling magnetic features on the photosphere, the photospheric reconnection seems to be one of the cancellation mechanisms.

Since the reconnection occurs in a stratified atmosphere, the upward and downward reconnection jets are not sym- 


\section{Temperature}

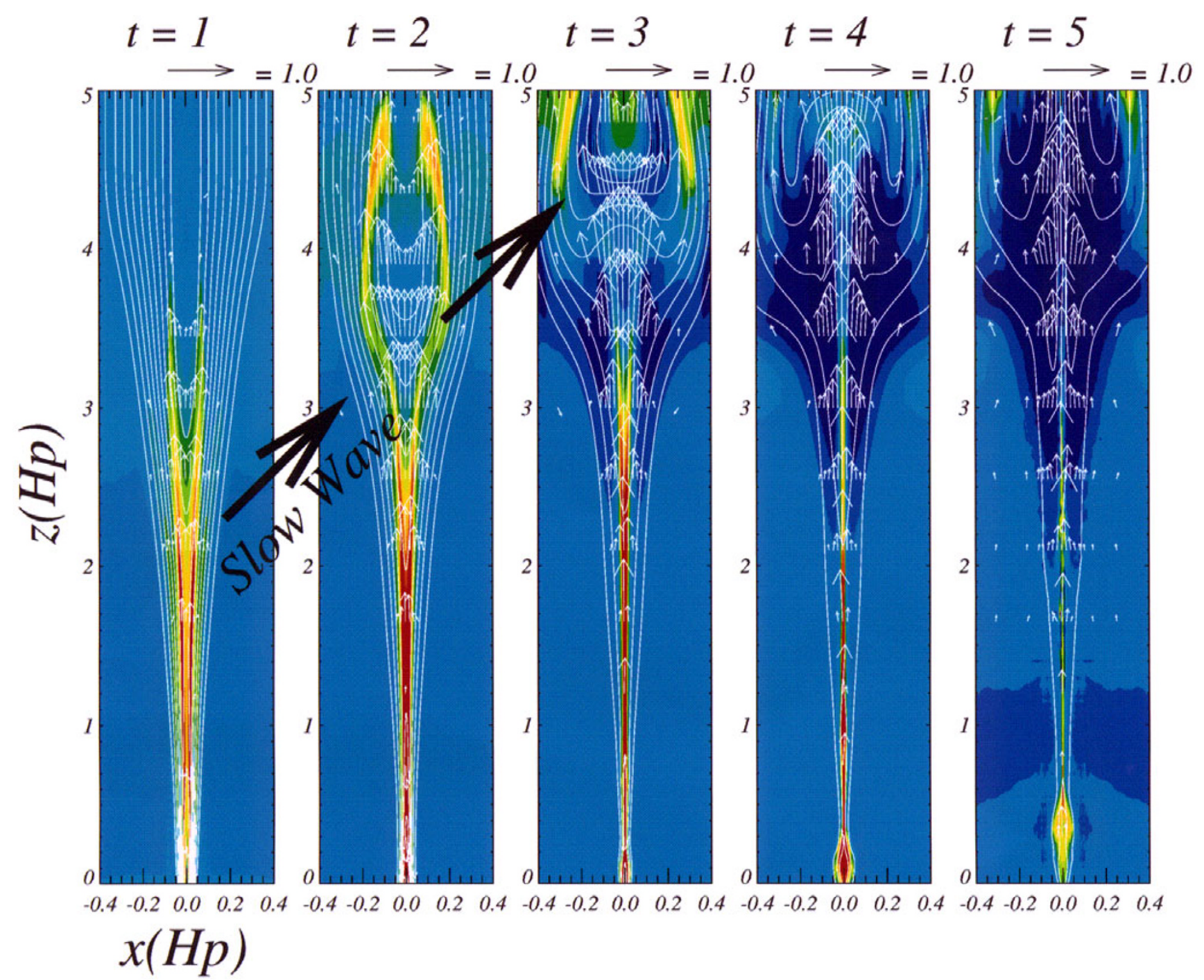

Fig. 2. Temporal evolution of temperature distribution due to the reconnection, when $\theta$ is taken to be $0^{\circ}$. The horizontal and vertical directions are $x$ and $z$ directions, respectively. The unit of time and temperature are $H_{p} / C_{s}(=20 \mathrm{~s})$ and the initial photospheric temperature $T_{0}(=6300 \mathrm{~K})$, respectively. Since the resistivity possesses a maximum at $z=2$, magnetic reconnection occurs in the neighborhood of the maximum $(t=1)$. The reconnection is the Sweet-Parker type (Sweet, 1958; Parker, 1963). MHD slow mode waves are generated by an upward reconnection jet, and they propagate upward along the magnetic field lines (Arrows show the propagation).

metric. It should be noted that slow mode MHD waves are generated by an upward reconnection jet. The slow waves propagate upward along the magnetic field lines. The propagation of the wave fronts are shown in Fig. 2 with arrows. As mentioned in Section 2, it seems unrealistic to assume that the initial flux sheets are perfectly anti-parallel. We next investigate effects of the inclination angle $\theta$ upon the reconnection. In Fig. 3 we show the temporal evolution of the $y$-component of magnetic field $B_{y}$, where $\theta=30^{\circ}$. It is apparent from the figure that $B_{y}$ is injected by the reconnection into the right flux sheet, where initially $B_{y}$ is 0 , and the injected $B_{y}$ propagates as Alfvén waves along field lines.

In the models of solar spicules upward propagating slow waves or Alfvén waves are usually assumed as initial per- turbations (Hollweg et al., 1982; Suematsu et al., 1982; Hollweg, 1982; Sterling and Mariska, 1990; Hollweg, 1992; Kudoh and Shibata, 1999). It is interesting to compare the wave energies with those generated by the reconnections. The energy flux carried by the waves generated by reconnection are measured at $z=5(=800 \mathrm{~km})$ in the following way.

$$
\begin{aligned}
F_{\text {slow }} & =\frac{1}{L} \int_{x_{0}}^{x_{1}} \Delta p v_{z} d x, \\
F_{\text {Alfvén }} & =-\frac{1}{4 \pi L} \int_{x_{0}}^{x_{1}} B_{y} v_{y} B_{z} d x,
\end{aligned}
$$

where $F_{\text {slow }}$ and $F_{\text {Alfvén }}$ are the energy flux of the slow and Alfvén waves, respectively, $x_{0}$ and $x_{1}$ are the positions of the 


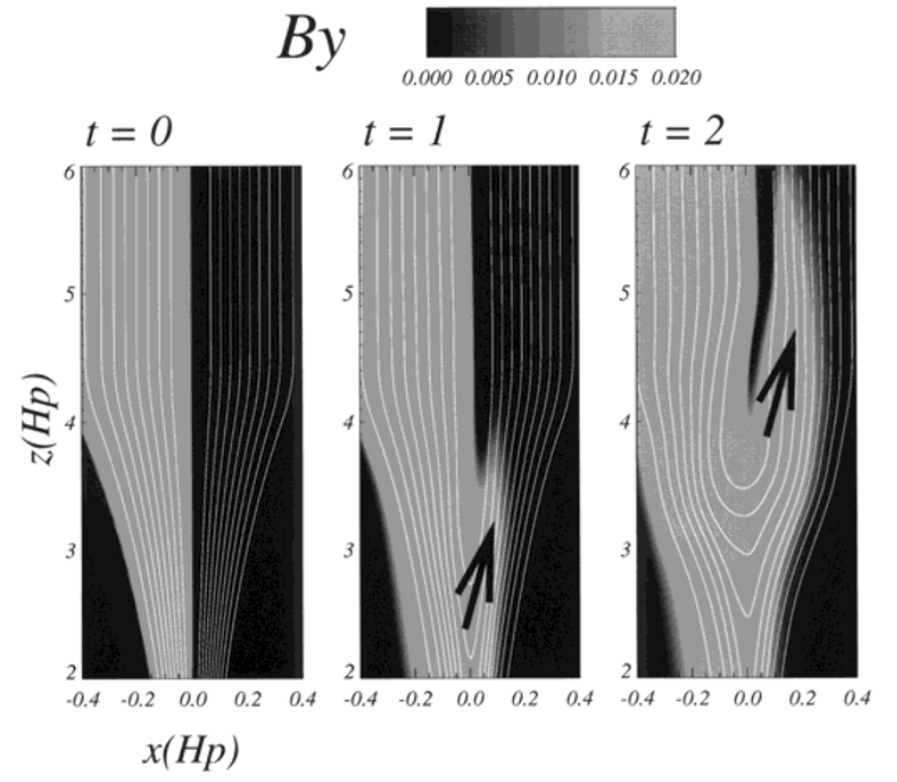

Fig. 3. Temporal evolution of $B_{y}$ due to magnetic reconnection, where $\theta=30^{\circ} . B_{y}$ is injected into the right half part, and propagates as Alfvén waves along field lines.

center and the right side of the flux sheet, respectively, and $L=x_{1}-x_{0}$, and $\Delta p=p(t)-p(0)$.

In Fig. 4 we show temporal evolution of the energy flux where the slow and Alfvén waves are shown as dashed and solid curves, respectively. The energy flux is normalized to $P_{0} C_{S}\left(\sim 10^{11} \mathrm{erg} / \mathrm{cm}^{2} / \mathrm{s}\right)$ where $P_{0}$ is the photospheric gas pressure. Figure 4 shows that the energy fluxes generated by the reconection are $F_{\text {slow }} \sim 10^{9} \mathrm{erg} / \mathrm{cm}^{2} / \mathrm{s}$ and $F_{\text {Alfvén }} \sim 10^{7} \mathrm{erg} / \mathrm{cm}^{2} / \mathrm{s}$, and the durations of these waves are $\tau_{\text {slow }} \sim \tau_{\text {Alfvén }} \sim 40 \mathrm{~s}$. Note that we measure the energy flux at $z=5$ where the width of the flux sheet is ten times wider than that at $z=0$. On the other hand, in many spicule models these waves are generated at the photospheric base. To compare the two energy fluxes, we multiply the energy flux generated by the reconnections by 10 . Thus, if we transform the flux into those at the photosphere, $F_{\text {slow }} \sim 10^{10}$ $\mathrm{erg} / \mathrm{cm}^{2} / \mathrm{s}$ and $F_{\text {Alfvén }} \sim 10^{8} \mathrm{erg} / \mathrm{cm}^{2} / \mathrm{s}$. In the spicule models of Sterling and Mariska (1990) $F_{\text {slow }} \sim 5 \times 10^{8} \mathrm{erg} / \mathrm{cm}^{2} / \mathrm{s}$ and $\tau_{\text {slow }} \sim 100 \mathrm{~s}$ and of Hollweg (1992) $F_{\text {Alfvén }} \sim 3 \times 10^{9}$ $\mathrm{erg} / \mathrm{cm}^{2} / \mathrm{s}$ and $\tau_{\text {Alfvén }} \sim 50 \mathrm{~s}$. Although the energy flux of the slow waves seems large enough, the Alfvén flux is not enough to produce that seen in solar spicules. In our calculations we have adopted $\beta=0.3$ as the minimum value in the initial flux sheet. In the actual photospheric flux tubes, however, 0.3 is the mean value of $\beta$ (Solanki, 1993). Thus, the minimum $\beta$ might be smaller than 0.3 and as we decrease the minimum $\beta$ the Alfvén flux increases. For this reason, the wave energies generated by reconnection could be comparable to those assumed in the spicule models.

In our calculations, ionization, radiative relaxation, and heat conduction are omitted. The generation of the Alfvén waves is not sensitive to these omissions. Also, the omission of ionization does not affect the generation of the slow waves. Since, in the actual solar atmosphere, the reconnection takes place in the temperature-minimum region where the dynamical time scale $H_{p} / C_{S}(=20 \mathrm{~s})$ is much shorter

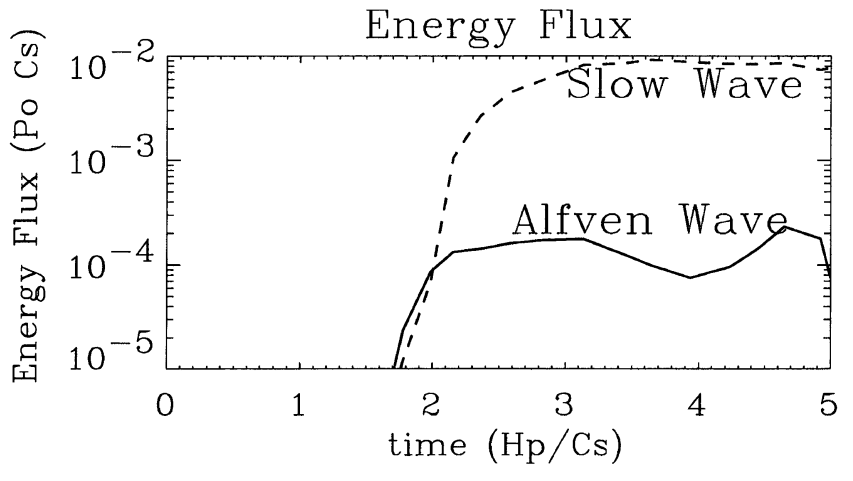

Fig. 4. Temporal evolution of the energy flux where the slow modes and Alfvén waves are shown as dashed and solid curves, respectively. The energy flux is normalized with $P_{0} C_{s}\left(\sim 10^{11} \mathrm{erg} / \mathrm{cm}^{2} / \mathrm{s}\right)$ where $P_{0}$ is the photospheric gas pressure. The energy flux generated by the reconections are $F_{\text {slow }} \sim 10^{9} \mathrm{erg} / \mathrm{cm}^{2} / \mathrm{s}$ and $F_{\text {Alfvén }} \sim 10^{7} \mathrm{erg} / \mathrm{cm}^{2} / \mathrm{s}$, and the durations are $\tau_{\text {slow }} \sim \tau_{\text {Alfvén }} \sim 40 \mathrm{~s}$.

than the radiative relaxation time $\left(\sim 10^{2} \mathrm{~s}\right)$, the generation of the slow waves is not affected by the neglect of non-adiabatic effects. Thus, since the wave energies generated by the reconnection process are comparable to those assumed in the spicule models, photospheric magnetic reconnection might be one of the causes of solar spicules.

\section{Conclusions}

We perform 2.5-dimensional MHD numerical simulations to investigate photospheric magnetic reconnection caused by an encounter of oppositely directed vertical magnetic flux sheets. We calculate the initial flux sheets adopting the thin flux tube approximation. In the simulations the resistivity is assumed a function of height with a maximum (where $R_{m}=1000$ ) at the middle height of our simulation box to simulate the actual solar resistivity.

The main results are summarized as follows: 
1) Sweet-Parker type magnetic reconnection occurs at the height where the resistivity possesses a maximum (temperature-minimum region).

2) Since the inflow speed of the reconnection $\left(v_{i} \sim 160\right.$ $\mathrm{m} / \mathrm{s}$ ) is nearly equal to the speeds implied by observations of canceling magnetic features on the photosphere, the photospheric reconnection seems to be one of the cancellation mechanisms.

3) Upward propagating MHD slow mode waves and Alfvén waves are generated by the magnetic reconnection. The energy flux are $F_{\text {slow }} \sim 10^{10} \mathrm{erg} / \mathrm{cm}^{2} / \mathrm{s}$ and $F_{\text {Alfvén }} \sim 10^{8} \mathrm{erg} / \mathrm{cm}^{2} / \mathrm{s}$ and the durations are $\tau_{\text {slow }} \sim$ $\tau_{\text {Alfvén }} \sim 40 \mathrm{~s}$ at the photosphere.

4) In models of solar spicules, upward propagating slow waves or Alfvén waves are usually assumed as initial perturbations. The wave energies due to the reconnections are comparable to those assumed in the spicule models. The photospheric magnetic reconnection might be one of the important causes of solar spicules.

Acknowledgments. The authors thank Dr. T. Kudoh, Dr. T. Yokoyama, and Dr. S. Tanuma for useful discussions. Numerical computations were carried out on VX/1R at National Astronomical Observatory, Japan.

\section{References}

Garcia de la Rosa, J. I., M. A. Aballe, and M. Collados, An example of the cancellation of magnetic fields during the decay of an active region, Sol. Phys., 124, 219-226, 1989.

Hollweg, J. V., On the origin of solar spicules, ApJ, 257, 345-353, 1982.

Hollweg, J. V., Alfvénically driven slow shocks in the solar chromosphere and corona, ApJ, 389, 731-738, 1992.

Hollweg, J. V., S. Jackson, and D. Galloway, Alfvén waves in the solar atmosphere III. Nonlinear waves on open flux tubes, Sol. Phys., 75, 35-61, 1982.

Kovitya, P. and L. Cram, Electrical conductivity in sunspots and the quiet photosphere, Sol. Phys., 84, 45-48, 1983.

Kudoh, T. and K. Shibata, Alfvén wave model of spicules and coronal heating, ApJ, 514, 493-505, 1999.

Litvinenko, K., Photospheric magnetic reconnection and canceling magnetic features on the Sun, ApJ, 515, 435-440, 1999.

Parker, E. N., The solar-flare phenomenon and the theory of reconnection and annihilation of magnetic fields, ApJS, 8, 177-211, 1963.

Richtmyer, R. D. and K. W. Morton, Difference Method for Initial Value Problem, 2nd ed., 401 pp., Interscience Publishers, New York, 1967.

Roberts, B. and A. R. Webb, Vertical motions in an intense magnetic flux tube, Sol. Phys., 56, 5-35, 1978.

Rubin, E. L. and S. Z. Burstein, Difference methods for the inviscid and viscous equations of a compressible gas, J. Comp. Phys., 2, 178-196, 1967.

Schrijver, C. J., A. M. Title, A. A. van Ballegooijen, and R. A. Shine, Sustaining the quiet photospheric network: The balance of flux emergence, fragmentation, merging, and cancellation, ApJ, 487, 424-436, 1997.

Schrijver, C. J., A. M. Title, K. L. Harvey, N. R. Sheeley, Jr., Y.-M. Wang, G. H. J. van den Oord, R. A. Shine, T. D. Tarbell, and N. E. Hurlburt, Large-scale coronal heating by the small-scale magnetic field of the Sun, Nature, 394, 152-154, 1998.

Solanki, S. K., Small scale solar magnetic fields: An overview, Space Sci. Rev., 61, 1-188, 1993.

Sterling, A. C. and J. T. Mariska, Numerical simulations of the rebund shock model for solar spicules, ApJ, 349, 647-655, 1990.

Sturrock, P. A., Chromospheric magnetic reconnection and its possible relationship to coronal heating, ApJ, 521, 451-459, 1999.

Suematsu, Y., K. Shibata, T. Nishikawa, and R. Kitai, Numerical hydrodynamics of the jet phenomena in the solar atmosphere I. Spicules, Sol. Phys., 75, 99-118, 1982.

Sweet, P. A., The neutral point theory of solar flares, in IAU Symp. 6, Electromagnetic Phenomena in Cosmical Physics, edited by B. Lehnert, pp. 123-134, Cambridge Univ. Press, Cambridge, 1958.

Takeuchi, A. and K. Shibata, Magnetic reconnection induced by convective intensification of solar photospheric magnetic fields, ApJL, 546, L73L76, 2001.

A. Takeuchi (e-mail: takeuchi@yonago-k.ac.jp) and K. Shibata 